\title{
Supplementary Material: Total Internal Reflection Peak Force Infrared Microscopy
}

Haomin Wang ${ }^{1}$, Le Wang ${ }^{1}$, Eli Janzen ${ }^{2}$, James H. Edgar ${ }^{2}$, and Xiaoji G. Xu ${ }^{1 *}$

${ }^{1}$ Department of Chemistry, Lehigh University, Bethlehem, PA 18015, USA

${ }^{2}$ Tim Taylor Department of Chemical Engineering, Kansas State University, Durland Hall, Manhattan, KS 66506, USA

*Corresponding email: xgx214@1ehigh.edu

This supplementary material includes:

Figure S1. Polymer thickness measurements. 
(a)
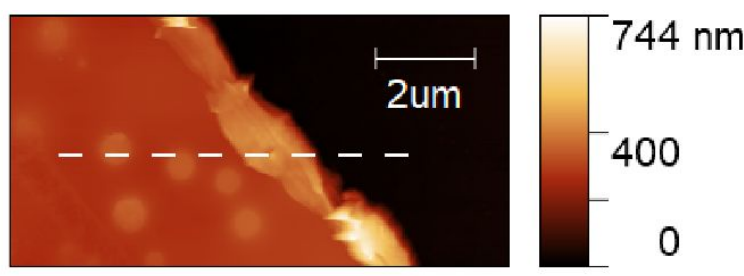

(c)

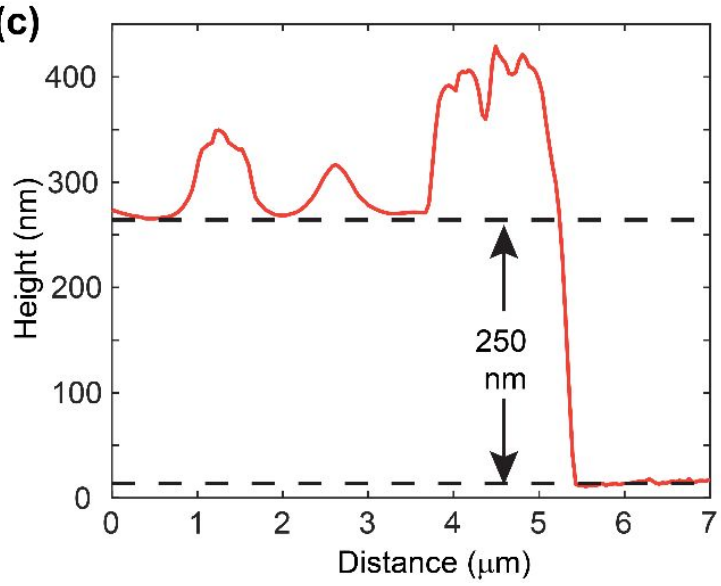

(b)

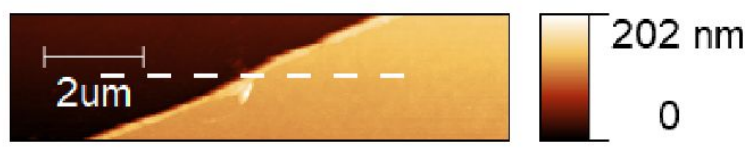

(d)

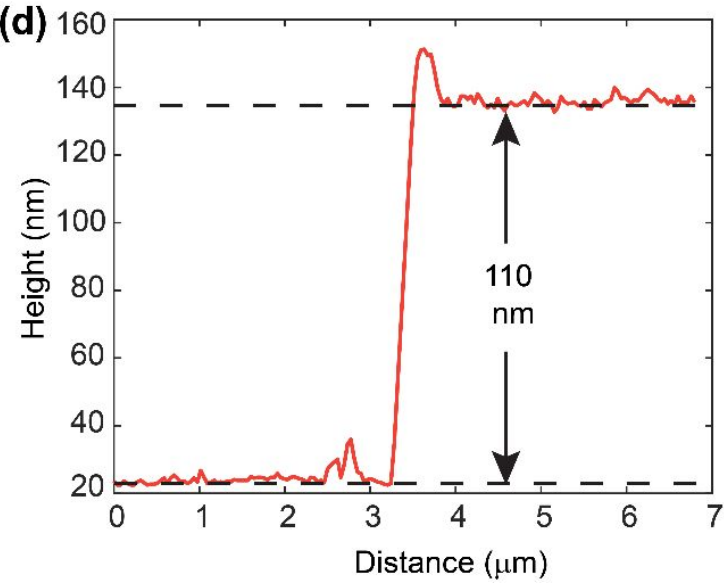

Figure S1. Polymer thickness measurements. (a-b) Topography images of PS:PMMA blend film and PS-b-PMMA co-block polymer film measured in the main text, respectively. Grooves are artificially made on polymer films to expose Ge surface and enable the thickness measurement. (c-d) Signal profiles obtained from dashed lines in (a) and (b), respectively. In (c), a thickness of $250 \mathrm{~nm}$ is obtained for the PS:PMMA blend film. In (d), a thickness of $110 \mathrm{~nm}$ is obtained for the PS-b-PMMA co-block polymer film. 